\title{
Experiments
}

\section{Apparatus and starting material}

Experiments have been performed at $2.5 \mathrm{GPa}$ and at $800^{\circ} \mathrm{C}$ and $850^{\circ} \mathrm{C}$ on a representative gneiss from Drage, Stadlandet (Table 1). Experiments were conducted in a 1/2 inch pistoncylinder apparatus using talc-pyrex-graphite furnace assemblies and gold capsules. The use of these assemblies and gold capsules produce conditions around the Ni-NiO transition. Run durations were between 72-137 hours, depending on run conditions. The gneiss has an initial mineralogy of mainly quartz, biotite, phengite, garnet and plagioclase. We conducted the experiments using directly the powdered gneiss, which has $~ 1.74 \mathrm{wt} \% \mathrm{H}_{2} \mathrm{O}$ bound in hydrous minerals. We added 5 or $10 \mathrm{wt} \% \mathrm{H}_{2} \mathrm{O}$ to the experimental charges.

\section{Analytical techniques}

Capsules mounted in epoxy resine were polished for scanning electron microscopy (SEM) and electron microprobe analyses. Major elements of glasses were analysed with the following analytical conditions, accelerating voltage of $15 \mathrm{kv}$, sample current of $6 \mathrm{nA}$, counting time of $10 \mathrm{~s}$ on peak, and a defocused beam $(10 \mu \mathrm{m})$. The $\mathrm{H}_{2} \mathrm{O}$ contents of quenched glasses were determined using the by-difference method calibrated with a set of 4 rhyolitic hydrous glass standards whose $\mathrm{H}_{2} \mathrm{O}$ contents (0-6.38 wt\%) have been determined by either ion microprobe analysis or Karl Fisher titration (Scaillet et al., 1995; Martel et al., 1998; Scaillet and Evans, 1999).

Martel, C., Pichavant, M., Bourdier, J.L., Traineau, H., Holtz, F., and Scaillet, B., 1998, Magma storage conditions and control of eruption regime in silicic volcanoes : experimental evidence from Mt. Pelée: Earth and Planetary Science Letters, v. 156, p. 89-99.

Scaillet, B., and Evans, B.W., 1999, The June 15, 1991 eruption of Mount Pinatubo. I. Phase equilibria and pre-eruption P-T-fO2-fH2O conditions of the dacite magma: Journal of Petrology, v. 40, p. 381-411.

Scaillet, B., Pichavant, M., and Roux, J., 1995, Experimental crystallisation of leucogranite magmas: Journal of Petrology, v. 36, p. 663-705. 
Results

\begin{tabular}{|c|c|c|c|c|}
\hline Sample/Glass & NOG004-1 & $800 / 5$ & $850 / 5$ & $800 / 10$ \\
\hline${\text { Experiment } n^{\circ}}^{\circ}$ & & norWM1 & norWM3 & norWM5 \\
\hline P (Gpa) & & 2.5 & 2.5 & 2.5 \\
\hline $\mathbf{T}\left({ }^{\circ} \mathbf{C}\right)$ & & 800 & 850 & 800 \\
\hline Added H2O (wt\%) & & 5 & 5 & 10 \\
\hline \multicolumn{5}{|c|}{ \%fluid phase in exp. } \\
\hline Major phases & & gl, grt, bi, qtz, ky & gl, grt, qtz, ky & gl, grt, bi, qtz, ky \\
\hline Accessory phases & & rt, sa, ap, zr, plg & rt, alb, bi,cpx & rt, s, st, zr \\
\hline \multicolumn{5}{|c|}{ Major elements (wt\%) } \\
\hline n Analyses & & 18 & 16 & 19 \\
\hline $\mathrm{SiO2}$ & 68.53 & 72.57(39) & $74.65(67)$ & $74.82(49)$ \\
\hline TiO2 & 0.74 & $0.26(7)$ & $0.25(4)$ & $0.19(4)$ \\
\hline Al2O3 & 14.07 & $15.87(27)$ & $14.64(53)$ & $15.20(25)$ \\
\hline FeO & 5.63 & $0.56(13)$ & $1.42(15)$ & $1.36(14)$ \\
\hline MnO & 0.07 & $0.03(5)$ & $0.04(7)$ & $0.04(4)$ \\
\hline MgO & 2.55 & $0.34(6)$ & $0.68(7)$ & $0.65(9)$ \\
\hline $\mathrm{CaO}$ & 1.21 & $0.92(10)$ & $1.04(9)$ & $1.31(13)$ \\
\hline Na2O & 2.22 & $5.41(29)$ & $2.69(32)$ & $2.45(37)$ \\
\hline K2O & 3.32 & $3.90(27)$ & $4.41(26)$ & $3.80(14)$ \\
\hline Hydrous Total & 100.25 & $92.05(55)$ & $88.95(73)$ & 87.39(73) \\
\hline H2O & & $6.16(54)$ & $9.05(71)$ & 10.51(69) \\
\hline
\end{tabular}

a sulphide

b alumino-silicate

Table 1 : Starting material composition and experimental glasses mineralogy and compositions. Partial melting rates were estimated by least square mass balance calculation above $38 \%$ for all experiments.

Experimental textures

- File fig1suppmat attached aside -

Figure 1 : back-scattered electron images of experimental result for NorWM5 at $800^{\circ} \mathrm{C}$ and $10 w t \%$. Bi1 and Bi2 respectively represent biotites with compositions similar and different from the starting material biotites. 


\section{Natural leucosomes}

\begin{tabular}{|c|c|c|c|c|c|c|c|c|c|}
\hline Location & $\begin{array}{l}\text { NO10-16 } \\
\text { Flugevåg }\end{array}$ & $\begin{array}{c}\text { NO10-23 } \\
\text { Haugsbygda }\end{array}$ & $\begin{array}{c}\text { NO10-27 } \\
\text { Haugsbygda }\end{array}$ & $\begin{array}{c}\text { NO10-43 } \\
\text { Fjørtoft }\end{array}$ & $\begin{array}{c}\text { NO10-45-1 } \\
\text { Midsund }\end{array}$ & $\begin{array}{c}\text { NO10-45-2 } \\
\text { Midsund }\end{array}$ & $\begin{array}{c}\text { NO10-51 } \\
\text { Bud }\end{array}$ & $\begin{array}{c}\text { NO10-55 } \\
\text { Svartberget }\end{array}$ & $\begin{array}{c}\text { NO10-60 } \\
\text { Sjøholt }\end{array}$ \\
\hline Lat. $\mathbf{N}$ & $62^{\circ} 07.921^{\prime}$ & $62^{\circ} 15.495^{\prime}$ & $62^{\circ} 15.495^{\prime}$ & $62^{\circ} 42.759^{\prime}$ & $62^{\circ} 39.902^{\prime}$ & $62^{\circ} 39.902^{\prime}$ & $62^{\circ} 41.303^{\prime}$ & $62^{\circ} 50.973^{\prime}$ & $62^{\circ} 28.675^{\prime}$ \\
\hline Long. E & $005^{\circ} 24.456$ & $005^{\circ} 31.941^{\prime}$ & $005^{\circ} 31.941^{\prime}$ & $006^{\circ} 25.467$ & $006^{\circ} 41.030$ & $006^{\circ} 41.030$ & $006^{\circ} 50.085$ & $007^{\circ} 00.351^{\prime}$ & $006^{\circ} 44.385$ \\
\hline $\mathrm{SiO} 2$ & 74.91 & 64.75 & 69.77 & 74.23 & 74.53 & 74.67 & 69.62 & 75.55 & 74.16 \\
\hline Al2O3 & 12.92 & 19.72 & 15.54 & 14.44 & 15.17 & 14.47 & 11.52 & 14.38 & 13.75 \\
\hline $\mathrm{Fe} 2 \mathrm{O} 3$ & 1.66 & 0.17 & 1.27 & 0.54 & 0.13 & 0.54 & 4.75 & 0.96 & 0.68 \\
\hline MnO & 0.03 & 0.01 & 0.02 & 0.03 & 0.00 & 0.02 & 0.06 & 0.03 & 0.01 \\
\hline MgO & 0.29 & 0.02 & 0.23 & 0.06 & 0.05 & 0.10 & 2.72 & 0.28 & 0.09 \\
\hline $\mathrm{CaO}$ & 1.22 & 1.91 & 0.89 & 1.34 & 1.66 & 1.46 & 5.18 & 1.18 & 1.26 \\
\hline Na2O & 2.31 & 5.90 & 2.96 & 4.11 & 4.90 & 4.24 & 2.81 & 2.78 & 2.98 \\
\hline K2O & 5.75 & 6.30 & 8.30 & 4.48 & 3.08 & 4.16 & 0.95 & 3.49 & 5.44 \\
\hline TiO2 & 0.13 & 0.02 & 0.15 & 0.04 & 0.02 & 0.06 & 0.67 & 0.08 & 0.05 \\
\hline P2O5 & 0.06 & $<$ d. 1. & $<$ d. 1. & $<$ d. 1. & $<$ d. l. & $<$ d. 1. & 0.16 & 0.07 & $<$ d. 1. \\
\hline loi & 0.48 & 0.78 & 0.75 & 0.49 & 0.64 & 0.52 & 1.03 & 1.41 & 0.78 \\
\hline Total & 99.76 & 99.57 & 99.88 & 99.75 & 100.17 & 100.23 & 99.48 & 100.20 & 99.20 \\
\hline
\end{tabular}

Table 2 : major elements analysis of natural leucosomes sampled in the WGR. 


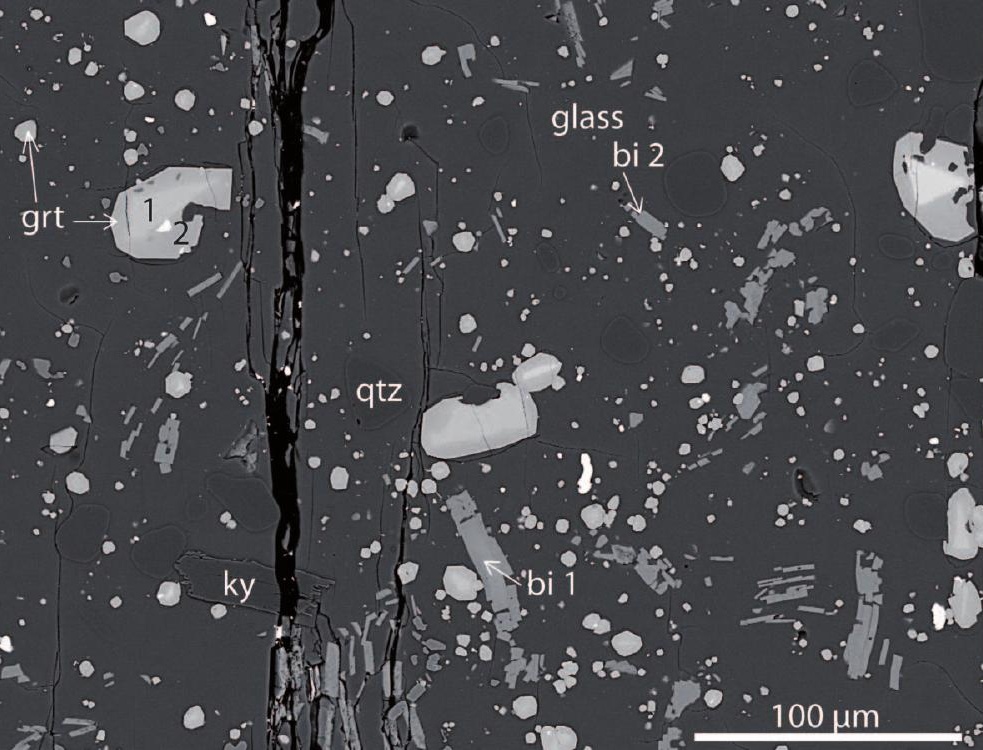

